\title{
Comparative Study of Different Preparations of Recombinant Insulin from Yeast Cells on STZ-Induced Insulin Dependent Diabetes Mellitus in Albino Rats
}

\author{
Suzan F.I. Elsisi*and Elgamal A.M** \\ * Physiology Department, NODCAR, Giza, Egypt \\ $* *$ Chemistry of Natural and Microbial Products, National Research Center
}

\begin{abstract}
Background: This study is used to compare the effects between three different INSUGEAST preparations (preps) (recombinant insulin from yeast cells) in management of insulin dependent diabetes mellitus (IDDM) and its complications. The preps were extracted from modified genetically saccharomyces cerviciae on culture pure camel milk (PC), partial pure camel milk (PPC) and specific media (SP) parallel with insulin stander (ST). Also the study extended to evaluate the difference in effect of the aforementioned INSUGEAST preps in three forms, regular, biphasic and recombinant in yeast cells against the stability and severity of hyperglycemia. Method: IDDM was induced in female albino rats by intraperitoneal injection (i.p) of a single dose $70 \mathrm{mg} / \mathrm{kg} / \mathrm{B}$. wt of streptozotocin (STZ) in citrate buffer. The diabetic rats were classified into two groups; the $1^{\text {st }}$ group divided into 5 equal subgroups, four subgroups, each was injected subcutaneously (s.c) by PC, PPC, SP and St respectively, in the biphasic form, in a dose of $4.2 \mathrm{IU} / \mathrm{kg} / \mathrm{B} . \mathrm{wt}$ for 3 weeks.The $5^{\text {th }}$ subgroup was considered as the diabetic control (STZ), in addition to 6 normal rats was used as free control group $(C)$. The $2^{\text {nd }}$ group divided as the same as in $1^{\text {st }}$ group but the INSUGEAST preps used involve little protamine zinc or in the regular form in addition to other 2 subgroups, each of them treated orally either with an amount of yeast $(Y)$ containing insulin equivalent to $4.2 \mathrm{IU} / \mathrm{kg} / \mathrm{B} . \mathrm{wt}$ or added to it specific additive (YA) and treated for the same period. Blood glucose level either fasting (FBG) or after $2 \mathrm{~h}$ from glucose and insulin loading were measured weekly for all groups. Biochemical assays to ascertain diabetic complications resemble in hepatorenal dysfunction included: aminotransferase aspartate (AST) and alanate (ALT), alkaline phosphatase (ALP), creatinine (Creat) and blood urea nitrogen (BUN); hyperlipidemia indicators included: total cholesterol (T-Ch), low density lipoprotein cholesterol (LDL-Ch), high density lipoprotein cholesterol (HDL-Ch) and triglycerides (TG) were measured at the end of the experimental schedule. The result of this study showed that: 1 - Blood glucose level in $1^{\text {st }}$ group was markedly decreased after insulin injection in all different preps and similar to the effect of insulin standard. 2- The hepato-renal dysfunction and hyperlipidemea induced by STZ injection, showed significant improvements after INSUGEAST preps (biphasic form), especially with insulin prepared on PPC and PC media. 3- INSUGEAST preps on the $2^{\text {nd }}$ group (regular form, in the yeast) decrease the level of FBG significantly only at $1^{\text {st }}$ week of treatment, their reduction effect decrease, the severity of hyperglycemia
\end{abstract}


increase, animals could not survive and mortality rate increase 4- The diabetic complications (hepato-renal dysfunction, hyperlipidemia) increased in all groups of this part and not showed any improvements. 5- Histopathological study made on pancreas, liver and kidney tissues conform the present finding. Conclusion: The INSUGEAT preps in biphasic form had the more potent effect in management of STZinduced IDDM and its complications. These effects were pronounced in PC and PPC preps and to a lesser extend in SP prep.

Key words: INSUGEAST preps, pure camel milk, partial pure camel milk, specific media, IDDM, STZ, diabetic complications.

\section{INTRODUCTION}

\author{
Diabetes is complex of \\ syndromes characterized
} metabolically by hyperglycemia, due to deficiency or diminished effectiveness of insulin. Insulin dependent diabetes mellitus (IDDM) is characterized by an apparently regular onset, a tendency to ketosis and absolute dependence on insulin to survive and maintain health ${ }^{[\mathbf{1 , 2}]}$. Lack of insulin secretion appear to result from an autoimmune process involving the pancreatic insulin producing cells leading to their destruction with development of diabetic syndrome $e^{[2,3]}$. They found that STZ could induce diabetes in experimental animals by direct damage of B-cells of the islet of Langerhans $^{[4,5]}$. Lack of insulin, whether absolute or relatives affects the metabolism of carbohydrate, protein, fats, water and electrolytes. It associated pathologically with specific microvascular complications plus various complications, including neuropathy, retinopathy, nephropathy, hepatic impairment and an increased susceptibility to infection ${ }^{[\mathbf{6}]}$. Numerous studies indicate that intensive therapy with insulin, which establishes normoglycemia in rats with diabetes, prevents these pathological complications induced in poorly controlled diabetic animals. It ameliorates impaired corneal reepithelialization in diabetic rats $^{[7]}$, prevents or corrects myelinated axon commitment by diabetes ${ }^{[8]}$. In contrast the recent study of Bixler, et al., $(2011)^{[9]}$, showed that chronic insulin treatment of diabetes does not fully normalize alterations in the retinal transcriptome. Moreover, the ultimate complication for diabetic patients, particularly in IDDM patients is hyperlipidemia with coronary heart diseases that are ameliorated by insulin treatment ${ }^{[1]}$.

The therapy of diabetes began with the use of pancreatic extract of beef to lower blood glucose level in pancreatomized dogs, nowadays; several preps of bovine and human insulin are available for therapy of IDDM. Although bovine and porcine insulin are similar to human insulin, their composition is slightly different. Consequently, a number of patients' immune systems produce antibodies against it, neutralizing its actions and resulting in inflammatory responses at injection sit, were fears of long term complications ensuing from the regular injection of a foreign substance, as well as a projected decline in the production of animal derived insulin. These factors led 
researchers to consider synthesizing Humulin by inserting the insulin gene into a suitable vector, the $E$. coli bacterial cell, to produce insulin that is chemically identical to its naturally produced counterpart. This has been achieved using Recombinant DNA technology ${ }^{[10]}$. Human insulin is supplied in a variety of forms in solution or suspension for injection, crystalline insulin may be prepared for therapeutic use by making solution either of acidic or neutral $\mathrm{pH}$ so called soluble insulin, rapid or regular insulin. In order to prolong the duration of action of insulin, the preparation involves either complexing insulin with protein, protamine zinc, from which it is slowly released or modifying the particle size as in various insulin zinc suspension $^{[11]}$, biphasic insulin is a mixture providing for both delayed and prolonged action ${ }^{[10]}$. Approximately 150 recombinant biopharmaceuticals have been approved by the Food and Drug Administration (FDA) and the list of submissions and approvals continues to grow $^{[12]}$. Our previous study used molecular biology techniques for insertion of human insugene into yeast, and INSUGEAST was extracted, purified and detected using HPLC. This study is the second part from the full paper and used to compare between the effects of three chosen INSUGEAST preps, prepared in three forms (regular, biphasic \& recombinant in yeast cells), in management of IDDM and its complications.

\section{MATERIALS \& METHODS}

\section{Materials}

- Streptozotocin (STZ); purchased from sigma with purity $98 \%$.

- INSUGEAST preps extracted from modified genetically saccharomyces cerviciae on culture pure camel milk, partial pure camel milk and specific media (produced in lab of Chemistry of Natural and Microbial Products - National Research Center.

- Purified standard insulin, purchased from lab of Chemistry of Natural and Microbial Products-National Research Center.

- Yeast cells containing recombinant insulin or recombinant insulin plus additive, prepared in lab of Chemistry of Natural Products and MicropyNational Research Center.

- Commercial available kits of BioMeriux, France, were purchased from Gama trade companyEgypt.

\section{Experimental design}

Animals

Adult female albino rats weighing $130 \pm 15 \mathrm{~g}$ were used. The animals were brought from laboratory animal breeding of National Organization for Drug Control and Research (NODCAR) Giza, Egypt. They were kept under strictly hygienic conditions. They were put on a standard basal diet and allowed free access to drinking water. The experimental protocols were approved by the NODCAR's Institutional Ethical Guidelines for Animal Care and Usage. 


\section{Induction of diabetes}

All animals except 6 rats kept as a free control rats, were injected i.p with $70 \mathrm{mg} / \mathrm{kg}$ B.wt of STZ dissolved in citrate buffer $\mathrm{pH} 4.5$, freshly prepared and injected to rats within 5 minutes of preparation. After 7 days of injection, blood samples were withdrawn from retro-orbital vein of the STZ-treated rats as well as those of free control group. Blood samples were collected, left to coagulate and serum was harvested. STZ-treated rats which showed fasting serum glucose concentration more than $350 \mathrm{mg} / \mathrm{dl}$ were included in this study. The STZ diabetic animals were divided into two main groups; the $1^{\text {st }}$ group used to test the effect of INSUGEAST preps in biphasic form on treatment of IDDM and its complications for 3 weeks as follow:

G1; Free not diabetic control group (C), fed on basal diet and injected with saline daily.

G2; STZ- diabetic control group (STZ); injected with STZ and left without treatment.

G3; insulin standard treated group (ST); injected subcutaneously with $4.2 \mathrm{IU} / \mathrm{kg}$ of ST daily.

G4,5,6; INSUGEAST treated groups (SP, PC, PPC); injected subcutaneously with $4.2 \mathrm{IU} / \mathrm{kg}$ of SP, PC, PPC respectively.

The $2^{\text {nd }}$ group of this experiment used to test the effect of INSUGEAST preps and insulin standard in regular form (involve little protamine zinc) on treatment of IDDM and its complications and subdivided as the same as in $1^{\text {st }}$ group in addition to other two diabetic subgroups, each of them treated orally either with an amount of yeast cells (Y) containing recombinant insulin or added to it specific additive (YA) for the same period as follow:

G7; yeast cells containing recombinant insulin treated group $(\mathrm{Y})$; orally administrated daily with

yeast cells containing amount of insulin equivalent to $4.2 \mathrm{IU} / \mathrm{kg}$.

G8; yeast cells containing recombinant insulin plus additive treated group (YA); orally adminis-

trated with yeast cells containing amount of insulin equivalent to $4.2 \mathrm{IU} / \mathrm{kg}$ plus additive.

At the end of the treatment schedule, blood samples were taken from each rat and then the animals were sacrificed, pancreas, liver and kidney tissues were removed and subjected to histopathological examinations as described by Bancrofet, et al. ${ }^{[13]}$.

\section{Biochemical techniques:}

Serum glucose was determined calorimetrically according to Trinder ${ }^{[14]}$, serum trasaminases (AST \& ALT) determined according to the method of Reitman and Frankel ${ }^{[15]}$, alkaline phosphates, BUN and Creat were determined by Roy ${ }^{[16]}$, Patton, and Cruoch ${ }^{[17]}$ and Houot ${ }^{[18]}$ respectively. Serum lipid profile include; serum triglycerides, total cholesterol, HDL-Cholesterol and LDL-Cholesterol were determined by Fossati and Prencipe ${ }^{[19]}$, Allain et al. ${ }^{[20]}$, Stein ${ }^{[21]}$ and Assmann et al..$^{[22]}$ respectively.

Statistical analysis: One-way ANOVA with determination of least significant difference was applied to study the relationship between the 
different variables, using SPSS version $11.5, \mathrm{P}<0.05$ was considered significant.

\section{RESULTS}

\section{Effect on blood glucose level:}

In the light of observation recoded in this work, table. 1 showed a significant decrease $(\mathrm{P}<0.05,86 \%)$ in the level of blood glucose after one week of treatment against hyperglycemia induced by STZ, the hypoglycemic effect was pronounced in PC and PPC groups and to a lesser extend in SP group while those treated with Y \& YA showed a lesser effect $(\mathrm{P}<0.05, \quad 9 \%$ and $19 \%$ glucose reduction respectively). The data showed that the hyperglycemic state of rats injected with INSUGEAST preps in biphasic form, showed marked improvement that increase with duration (table1). In conform, the light microscopic examination of pancreas of $1^{\text {st }}$ group, observed no alteration in the island of Langerhans cells except some congestion in the surrounding blood vessels in group of rats treated by PPC $\left(\right.$ Fig $\left.\mathrm{P}_{4}\right)$. While, in PC treatment, they showing intact histological structure of island of langerhans cells (Fig P5) against the dramatic effect showed in STZ diabetic rats (Fig $\mathrm{P}_{1,2,3}$ ). In contrast, rats treated with either regular form or yeast cells, the severity of hyperglycemia increase and potent activity of insulin for glucose reduction decreased to $30 \%$ with INSUGEAST preps and to $0 \%$ with yeast cells (table1). Also animals could not survive and mortality rate increase up to $60 \%$ during the last 2weeks of treatment (table 2).
Histopatholoical examination of the pancreas of these groups still showed atrophy in the cells of island and dilated ducts in addition to leucocytes inflammatory cells between them (Fig $\mathrm{P}_{6}$ ). On the other hand, groups of rats treated with ST, SP still showing some atrophy and depletion in the island of Langerhans cells.

\section{The effect on hepato-renal dysfunction:}

Regarding the effect of i.p injection of STZ to rats in this work, the results showed significant elevation $(\mathrm{P}<0.05)$ in the activities of liver enzymes (AST, ALT) as well as ALP compared to that observed in free control group (table $\left.3_{\mathrm{a} \& \mathrm{~b}}\right)$. Histopathological examination of rat liver injected with STZ showed marked cystic dilatation of the bile duct as well as periductal fibrosis, congestion in the central and portal veins (Fig $\mathrm{L}_{2}$ ). Diffuse kupffer cells proliferation with mononuclear leucocytes inflammatory cells infiltration was observed in between the hepatocytes (Fig $\mathrm{L}_{3}$ ), in respect to the normal structure observed in free control group (Fig $\mathrm{L}_{1}$ ). Contrary the STZ diabetic rats treated with INSUGEAST preps (biphasic form) showed significant improvement $(\mathrm{P}<0.05)$ of liver enzymes as well as in ALP $(\mathrm{P}<0.05)$ compared to that recorded in STZ treated group. Moreover, data showed that insulin standard induced decreases in liver enzymes in the STZ-diabetic rats similar to that induced by INSUGEAST preps (table 3). Furthermore the histopathological study on the liver of these groups showed mild changes (congestion in the central vein and sinusiods $\left(\mathrm{Fig}_{4}\right)$ 
and all histopathological changes noted in the untreated group were still present. On the other hand, rats treated with INSUGEAST preps in regular form showed significant elevation $(\mathrm{P}<0.05)$ of liver enzymes and ALP (table 3) and the histopathological changes were similar as in STZ diabetic group. In addition to focal necrosis $\left(\mathrm{FigL}_{5}\right)$ and fatty changes $\left(\right.$ FigL $\left._{6}\right)$ were found between the hepatocytes.

The analysis of blood urea and serum Creat in STZ diabetic rats showed marked increase $(\mathrm{P}<0.05)$ in both levels during period of experiment. In conform, the histopathological study of kidney removed from STZ diabetic rats showed congestion of the gloermular tuft of glomeruli with swelling in the lining endothelium (Fig $\mathrm{K}_{2}$ ), focal tubular degeneration and haemorrhages (Fig $\mathrm{K}_{3}$ ) in the corticomedullary junction, in respect to the normal structure observed in free control group (Fig $\left.\mathrm{K}_{1}\right)$. Treatment of STZ-diabetic rats with biphasic INSUGEAST especially PC showed significant improvement in kidney function $(\mathrm{P}<0.05)$ in comparison to the improvement effect of ST (table 3). The histopathological study showed mild changes (congestion in the cortical blood vessels (Fig K $\mathrm{K}_{4}$ ) but most the histopathological changes noted in the untreated group were still present. In contrast, impaired renal function were recorded in rats treated with INSUGEAST preps of rabid form (table 3) and the histopathological study of these groups still showed congestion of the glomerular tuft with swelling in the lining endothelium, focal tubular degeneration and hemorrhages in addition to focal suppurative embolus in the cortical portion $\left(\right.$ Fig $\left.\mathrm{K}_{5}\right)$ and focal fibrosis in the corticomedullary junction $\left(\right.$ Fig $\mathrm{K}_{6}$ ).

\section{Effect on hyperlipidemia:}

The data analysis showed marked increase $(\mathrm{P}<0.05)$ in the levels of $\mathrm{TG}$, $\mathrm{T}-\mathrm{Ch}$, and LDL-Ch accompanied with highly significant decrease in levels of HDL-Ch in STZ-diabetic rats. Treatment with biphasic INSUGEAST preps induced marked decrease $(\mathrm{P}<0.05)$ in all previous parameters except HDL-Ch which exhibit value higher $(\mathrm{P}<0.05)$ than recorded in STZ-diabetic group (table 4). Contrary, regular INSUGEAST preps could not improve the hyperlipidemic effect induced by STZ-diabetic group (table 4). 
Table (1): The effect of the three forms of the INSUGEAST preps on FBG (mg/dl) and after $2 \mathrm{~h}$ from their injection, of STZ-Diabetic rats

\begin{tabular}{|c|c|c|c|c|c|c|c|c|c|c|c|c|c|}
\hline \multirow{3}{*}{\multicolumn{2}{|c|}{$\begin{array}{r}\text { Animal } \\
\text { group } \\
\text { Duration }\end{array}$}} & \multirow{4}{*}{$\begin{array}{c}\begin{array}{l}\text { Normal } \\
\text { Control }\end{array} \\
\mathbf{C} \\
(n=10)\end{array}$} & \multirow{3}{*}{$\begin{array}{c}\begin{array}{c}\text { Diabetic } \\
\text { control }\end{array} \\
\text { STZ } \\
\end{array}$} & \multicolumn{10}{|c|}{ Treatment } \\
\hline & & & & \multicolumn{4}{|c|}{ Biphasic form } & \multicolumn{4}{|c|}{ Regular form } & \multicolumn{2}{|c|}{$\begin{array}{l}\text { Recombinant in yeast } \\
\text { cells }\end{array}$} \\
\hline & & & & ST & SP & PC & PPC & ST & SP & PC & PPC & $\mathbf{Y}$ & YA \\
\hline \multirow{5}{*}{$1^{\text {st }}$ wk } & & & $(\mathrm{n}=10)$ & $(\mathrm{n}=10)$ & $(n=10)$ & $(n=10)$ & $(n=10)$ & $(n=10)$ & $(n=10)$ & $(\mathrm{n}=10)$ & $(n=10)$ & $(n=10)$ & $(\mathrm{n}=10)$ \\
\hline & FBG & $95.29 \pm$ & $498.00 \pm$ & 505.85 & 520.00 & 511.25 & 514.83 & 525.17 & 558.00 & 533.17 & 547.83 & 500.17 & 561.17 \\
\hline & & 4.59 & $7.87^{*}$ & $\pm 9.77 *$ & $\pm 12.74^{*}$ & $\pm 7.75^{*}$ & $\pm 12.75^{*}$ & $\pm 19.62 *$ & $\pm 7.08^{*}, \mathrm{a}$ & $\pm 21.08^{*}$ & $\pm 12.45^{*}, \mathbf{a}$ & $\pm 25.70^{*}$ & $\pm 9.32^{*}, \mathbf{a}$ \\
\hline & & $(\mathrm{n}=10)$ & $(n=10)$ & $(n=10)$ & $(n=10)$ & $(n=10)$ & $(n=10)$ & $(n=10)$ & $(n=10)$ & $(n=10)$ & $(\mathrm{n}=10)$ & $(n=10)$ & $(n=10)$ \\
\hline & $2 \mathrm{~h}$ & $\begin{array}{l}92.85 \\
\pm 2.97\end{array}$ & $\begin{array}{c}490.00 \\
\pm 19.82^{*}\end{array}$ & $\begin{array}{c}70.83 \\
\pm 2.80^{*}, \mathbf{a} \\
(86 \%)\end{array}$ & $\begin{array}{c}75.66 \\
\pm 2.04^{*}, \mathrm{a} \\
(85 \%)\end{array}$ & $\begin{array}{c}73.67 \\
\pm 2.00^{*}, \mathbf{a} \\
(86 \%)\end{array}$ & $\begin{array}{c}70.00 \\
\pm 1.71^{*}, \mathbf{a} \\
(86 \%)\end{array}$ & $\begin{array}{l}67.83 \pm \\
3.95^{*, a} \\
(87 \%)\end{array}$ & $\begin{array}{c}69.00 \\
\pm 4.53^{*}, \mathbf{a} \\
(88 \%)\end{array}$ & $\begin{array}{c}73.83 \\
\pm 3.94^{*}, \mathbf{a} \\
(86 \%)\end{array}$ & $\begin{array}{c}72.50 \\
\pm 6.63^{*}, \mathrm{a} \\
(87 \%)\end{array}$ & $\begin{array}{c}453.00 \pm \\
31.41^{*, \mathbf{b . c}} \\
(9 \%)\end{array}$ & $\begin{array}{c}456.83 \pm \\
17.82^{*, b . c} \\
(19 \%)\end{array}$ \\
\hline \multirow{4}{*}{$2^{\text {nd }} w k$} & & $(n=10)$ & $(n=7)$ & $(n=10)$ & $(n=10)$ & $(n=10)$ & $(\mathrm{n}=10)$ & $(\mathrm{n}=8)$ & $(n=6)$ & $(n=6)$ & $(n=6)$ & $(\mathrm{n}=5)$ & $(n=5)$ \\
\hline & FBG & $\begin{array}{l}97.35 \\
\pm 3.85\end{array}$ & $\begin{array}{c}615.00 \\
\pm 19.18^{*}\end{array}$ & $\begin{array}{c}391.00 \\
\pm 7.23^{*}, \mathbf{a}\end{array}$ & $\begin{array}{c}445.67 \pm \\
16.50^{*, \mathbf{a}, \mathbf{b}, \mathbf{c}}\end{array}$ & $\begin{array}{c}418.48 \\
\pm 9.05^{*}, \mathbf{a b}\end{array}$ & $\begin{array}{c}384.74 \\
\pm 10.78^{*, a}\end{array}$ & $\begin{array}{l}658.80 \pm \\
15.23^{*, b}\end{array}$ & $\begin{array}{l}666.75 \pm \\
16.42^{*, \mathbf{b}}\end{array}$ & $\begin{array}{l}609.25 \pm \\
19.53^{*, \mathbf{c}, \mathbf{b}}\end{array}$ & $\begin{array}{c}540.25 \\
\pm 12.76^{*}, \mathrm{a}\end{array}$ & $\begin{array}{l}583.67 \pm \\
19.43^{*, \mathbf{c}}\end{array}$ & $\begin{array}{l}555.33 \pm \\
19.78^{*}, \mathbf{a , c} \mathbf{c}\end{array}$ \\
\hline & & $(\mathrm{n}=10)$ & $(n=7)$ & $(n=10)$ & $(n=10)$ & $(n=10)$ & $(n=10)$ & $(\mathrm{n}=8)$ & $(n=6)$ & $(\mathrm{n}=6)$ & $(\mathrm{n}=6)$ & $(n=5)$ & $(\mathrm{n}=5)$ \\
\hline & $2 h$ & $\begin{array}{l}98.82 \\
\pm 2.54\end{array}$ & $\begin{array}{c}580.3 \\
\pm 14.20^{*}\end{array}$ & $\begin{array}{l}66.63 \pm \\
2.58^{*}, \mathrm{a} \\
(83 \%)\end{array}$ & $\begin{array}{c}70.82 \\
\pm 2.36^{*}, \mathbf{a}, \mathbf{b} \\
(84 \%)\end{array}$ & $\begin{array}{c}62.25 \\
\pm 2.58^{*}, \mathbf{a} \\
(84 \%)\end{array}$ & $\begin{array}{c}61.39 \\
\pm 1.45^{*}, \mathbf{a} \\
(82 \%)\end{array}$ & $\begin{array}{c}457.60 \pm \\
22.19^{*}, \mathbf{a}, \mathbf{b} \\
(30 \%)\end{array}$ & $\begin{array}{c}435.5 \pm \\
19.84^{*}, \mathbf{a}, \mathbf{b} \\
(35 \%)\end{array}$ & $\begin{array}{c}366.75 \pm \\
26.99^{*}, \mathbf{a}, \mathbf{b}, \\
\mathbf{c}(40 \%)\end{array}$ & $\begin{array}{c}182.50 \pm \\
12.10^{*}, \mathbf{a , c} \\
(66 \%)\end{array}$ & $\begin{array}{c}585.67 \pm \\
18.35^{*}, \mathbf{b}, \mathbf{c} \\
(0 \%)\end{array}$ & $\begin{array}{c}509.67 \pm \\
18.35^{*, \mathbf{a}, \mathbf{b}} \\
(8 \%)\end{array}$ \\
\hline \multirow{5}{*}{$3^{\text {rd }} w k$} & & $(\mathrm{n}=10)$ & $(n=4)$ & $(n=10)$ & $(n=10)$ & $(n=10)$ & $(n=10)$ & $(\mathrm{n}=4)$ & $(\mathrm{n}=5)$ & $(\mathrm{n}=4)$ & $(\mathrm{n}=4)$ & $(\mathrm{n}=4)$ & $(n=4)$ \\
\hline & FBG & 98.22 & 598.00 & $349.00 \pm$ & $412.00 \pm$ & 387.00 & 338.00 & $654.50 \pm$ & 628.50 & 637.50 & 585.50 & 611.00 & 639.00 \\
\hline & & \pm 5.01 & $\pm 35.30^{*}$ & $17.41^{*}, \mathbf{a}$ & $10.55^{*, \mathbf{a}, \mathbf{b}, \mathbf{c}}$ & $\pm 16.55^{*}$,a,b & $\pm 10.48^{*}, \mathbf{a}$ & $19.50^{*}, \mathbf{b}$ & $\pm 21.54^{*}$ & $\pm 22.50^{*}$ & $\pm 20.50^{*}, \mathbf{c}$ & $\pm 18.00^{*}$ & $\pm 34.50 *$ \\
\hline & & $(\mathrm{n}=10)$ & $(n=4)$ & $(n=10)$ & $(\mathrm{n}=10)$ & $(\mathrm{n}=10)$ & $(n=10)$ & $(n=4)$ & $(n=4)$ & $(n=4)$ & $(n=4)$ & $(n=4)$ & $(n=4)$ \\
\hline & $2 \mathrm{~h}$ & $\begin{array}{l}94.48 \\
\pm 5.25\end{array}$ & $\begin{array}{c}584.17 \\
\pm 39.02 *\end{array}$ & $\begin{array}{l}60.63 \pm \\
2.61^{*}, \mathbf{a}\end{array}$ & $\begin{array}{c}65.36 \\
\pm 2.11^{*}, \mathrm{a}\end{array}$ & $\begin{array}{c}62.33 \\
\pm 2.11^{*}, \mathrm{a}\end{array}$ & $\begin{array}{c}60.67 \\
\pm 1.95^{*}, \mathrm{a}\end{array}$ & $\begin{array}{l}500.00 \pm \\
25.00^{*}, \mathbf{a}\end{array}$ & $\begin{array}{l}444.00 \pm \\
14.00^{*}, \mathbf{a}, \mathbf{b}\end{array}$ & $\begin{array}{c}446.51 \pm \\
38.50^{*}, \mathbf{a}, \mathbf{b}\end{array}$ & $\begin{array}{l}328.00 \\
\pm 5.00\end{array}$ & $\begin{array}{l}599.50 \pm \\
14.50^{*, \mathbf{b}, \mathbf{c}}\end{array}$ & $\begin{array}{l}585.50 \pm \\
15.50^{*}, \mathbf{b , c}\end{array}$ \\
\hline
\end{tabular}

- Significa 


\begin{tabular}{|c|c|c|c|c|c|c|c|c|}
\hline \multirow{2}{*}{$\begin{array}{l}\text { Groups } \\
\text { Duration }\end{array}$} & $\mathrm{C}$ & D & ST & SP & $\mathbf{C P}$ & $\mathbf{C A}$ & $\mathbf{Y}$ & YA \\
\hline & \multicolumn{8}{|c|}{ Number of animals per group and \% of mortality } \\
\hline Initial time & 6 & 10 & 10 & 10 & 10 & 10 & 10 & 10 \\
\hline $1^{\mathrm{ST}} \mathrm{wk}$ & $6(0 \%)$ & $10(0 \%)$ & $10 .(0 \%)$ & $10(0 \%)$ & $10(0 \%)$ & $10(0 \%)$ & $10(0 \%)$ & $10(0 \%)$ \\
\hline $2^{\text {nd }} w k$ & $6(0 \%)$ & $7(30 \%)$ & $8(20 \%)$ & $6(40 \%)$ & $6(40 \%)$ & $6(40 \%)$ & $5(50 \%)$ & $5(50 \%)$ \\
\hline $3^{\text {rd }}$ wk & $6(0 \%)$ & $4(60 \%)$ & $4(60 \%)$ & $4(60 \%)$ & $4(60 \%)$ & $4(60 \%)$ & $4(60 \%)$ & $4(60 \%)$ \\
\hline
\end{tabular}

Data represents number of animals per group and \% values of mortality from the initial time.

Table (3): The effect of the three forms of the INSUGEAST preps on hepato-renal dysfunctions of STZ-Diabetic rats

\begin{tabular}{|c|c|c|c|c|c|c|c|c|c|c|c|c|}
\hline \multirow{3}{*}{$\begin{array}{r}\begin{array}{l}\text { Animal } \\
\text { Groups }\end{array} \\
\text { Parameter }\end{array}$} & \multirow{3}{*}{$\begin{array}{c}\text { Normal } \\
\text { Control } \\
\mathrm{C} \\
\end{array}$} & \multirow{3}{*}{$\begin{array}{c}\text { Diabetic } \\
\text { Control } \\
\text { STZ }\end{array}$} & \multicolumn{10}{|c|}{ Treatment } \\
\hline & & & \multicolumn{4}{|c|}{ Biphasic form } & \multicolumn{4}{|c|}{ Regular form } & \multicolumn{2}{|c|}{$\begin{array}{l}\text { Recombinant in yeast } \\
\text { cells }\end{array}$} \\
\hline & & & ST & SP & PC & PPC & ST & SP & PC & PPC & $Y$ & YA \\
\hline $\begin{array}{c}\text { BUN } \\
(\mathrm{mg} / \mathrm{dl})\end{array}$ & $\begin{array}{l}34.50 \\
\pm 2.26\end{array}$ & $\begin{array}{r}93.50 \\
\pm 6.05^{*}\end{array}$ & $\begin{array}{c}74.83 \\
\pm 4.00^{*, a} \\
\end{array}$ & $\begin{array}{c}82.00 \\
\pm 6.05^{*, \mathbf{a , b}} \\
\end{array}$ & $\begin{array}{c}58.33 \\
\pm 5.01^{*, \mathbf{a}, \mathbf{b}, \mathbf{c}}\end{array}$ & $\begin{array}{c}46.00 \\
\pm 0.017^{*, a, c}\end{array}$ & $\begin{array}{c}91.73 \\
\pm 7.18^{*}\end{array}$ & $\begin{array}{c}103.13 \\
\pm 6.94 *\end{array}$ & $\begin{array}{c}84.17 \\
\pm 7.01 *\end{array}$ & $\begin{array}{l}84.17 \\
8.15^{*}\end{array}$ & $\begin{array}{c}83.67 \\
\pm 5.81 * \\
\end{array}$ & $\begin{array}{c}79.67 \\
\pm 4.96^{*} \\
\end{array}$ \\
\hline $\begin{array}{l}\text { AST } \\
(\mathbf{U} / \mathbf{L})\end{array}$ & $\begin{array}{r}95.67 \\
\pm 3.48\end{array}$ & $\begin{array}{c}184.67 \\
\pm 10.90^{*}\end{array}$ & $\begin{array}{c}139.17 \\
\pm 4.00^{*, a}\end{array}$ & $\begin{array}{c}149.83 \\
\pm 5.78^{*, a}\end{array}$ & $\begin{array}{c}147.83 \\
\pm 2.19^{*, a}\end{array}$ & $\begin{array}{c}137.36 \\
\pm 6.23^{*}, \mathrm{a}\end{array}$ & $\begin{array}{c}222.00 \\
\pm 12.33^{*, a}\end{array}$ & $\begin{array}{c}243.00 \\
\pm 13.18^{*, a}\end{array}$ & $\begin{array}{c}195.00 \\
\pm 12.85^{*}\end{array}$ & $\begin{array}{c}188.00 \\
\pm 12.97 *\end{array}$ & $\begin{array}{c}178.00 \\
\pm 10.85^{*}\end{array}$ & $\begin{array}{c}168.00 \\
\pm 13.97 *\end{array}$ \\
\hline $\begin{array}{l}\text { ALT } \\
(\mathbf{U} / \mathbf{L})\end{array}$ & $\begin{array}{l}54.00 \\
\pm 3.12\end{array}$ & $\begin{array}{l}101.00 \\
\pm 4.12 *\end{array}$ & $\begin{array}{c}87.83 \\
\pm 2.33^{*, a}\end{array}$ & $\begin{array}{c}98.83 \\
\pm 7.30^{*, a}\end{array}$ & $\begin{array}{c}91.67 \\
\pm 5.06^{*}\end{array}$ & $\begin{array}{c}91.50 \\
\pm 5.23^{*}\end{array}$ & $\begin{array}{c}117.00 \\
\pm 5.83^{*, a}\end{array}$ & $\begin{array}{c}124.00 \\
\pm 6.47^{*}, \text { a }\end{array}$ & $\begin{array}{c}114.00 \\
\pm 3.66^{*, a}\end{array}$ & $\begin{array}{l}107.00 \\
\pm 5.01 *\end{array}$ & $\begin{array}{c}133.00 \\
\pm 7.06^{*, a}\end{array}$ & $\begin{array}{c}123.00 \\
\pm 4.23^{*, \mathbf{a}}\end{array}$ \\
\hline $\begin{array}{l}\text { ALP } \\
(\mathbf{U} / \mathbf{L})\end{array}$ & $\begin{array}{l}40.67 \\
\pm 2.73\end{array}$ & $\begin{array}{l}109.00 \\
\pm 8.66^{*}\end{array}$ & $\begin{array}{c}63.50 \\
\pm 4.07^{*, a}\end{array}$ & $\begin{array}{c}78.83 \\
\pm 5.38^{*, \mathbf{a}, \mathbf{c}}\end{array}$ & $\begin{array}{c}68.33 \\
\pm 1.89^{*}, \mathrm{a}\end{array}$ & $\begin{aligned} & 69.67 \\
\pm & 2.29^{*}, \mathrm{a}\end{aligned}$ & $\begin{array}{l}115.00 \\
\pm 7.41^{*}\end{array}$ & $\begin{array}{l}122.00 \\
\pm 8.45^{*}\end{array}$ & $\begin{array}{l}117.52 \\
\pm 4.89^{*}\end{array}$ & $\begin{array}{c}115.74 \\
\pm 5.29 *\end{array}$ & $\begin{array}{c}127.96 \\
\pm 6.89^{*}\end{array}$ & $\begin{array}{l}117.00 \\
\pm 7.29 *\end{array}$ \\
\hline
\end{tabular}


Table (4): The effect of the three forms of the INSUGEAST preps on lipid parameters of STZ-Diabetic rats

\begin{tabular}{|c|c|c|c|c|c|c|c|c|c|c|c|c|}
\hline \multirow{3}{*}{$\begin{array}{r}\text { Animal } \\
\text { Groups } \\
\text { Parameter }\end{array}$} & \multirow{3}{*}{$\begin{array}{c}\text { Normal } \\
\text { Control } \\
\mathrm{C} \\
\end{array}$} & \multirow{3}{*}{$\begin{array}{c}\begin{array}{c}\text { Diabetic } \\
\text { control }\end{array} \\
\text { STZ } \\
\end{array}$} & \multicolumn{10}{|c|}{ Treatment } \\
\hline & & & \multicolumn{4}{|c|}{ Biphasic form } & \multicolumn{4}{|c|}{ Regular form } & \multicolumn{2}{|c|}{$\begin{array}{l}\text { Recombinant in } \\
\text { yeast cells }\end{array}$} \\
\hline & & & ST & SP & PC & PPC & ST & SP & PC & PPC & $\mathbf{Y}$ & YA \\
\hline $\begin{array}{c}\text { TG } \\
(\mathbf{m g} / \mathbf{d l})\end{array}$ & $\begin{array}{r}92.00 \\
\pm 5.13\end{array}$ & $\begin{array}{c}174.33 \\
\pm 3.41^{*}\end{array}$ & $\begin{array}{c}136.83 \\
\pm 4.65^{*, a} \\
\end{array}$ & $\begin{array}{c}147.17 \\
\pm 3.85^{*, \mathbf{a}, \mathbf{b}}\end{array}$ & $\begin{array}{c}130.00 \\
\pm 2.66^{*}, \mathrm{a} \\
\end{array}$ & $\begin{array}{c}134.83 \\
\pm 3.76^{*, a} \\
\end{array}$ & $\begin{array}{r}235.83 \\
\pm 5.70^{*, a}\end{array}$ & $\begin{array}{c}270.83 \\
\pm 10.81^{*, a, b, \mathbf{c}} \\
\end{array}$ & $\begin{array}{c}217.17 \\
\pm 10.81^{*}, \mathrm{a}\end{array}$ & $\begin{array}{c}217.50 \\
\pm 12.08^{*, a}\end{array}$ & $\begin{array}{c}213.83 \\
\pm 6.49^{*, a} \\
\end{array}$ & $\begin{array}{r}206.50 \\
\pm 9.65^{*, a} \\
\end{array}$ \\
\hline T-Ch (mg/dl) & $\begin{array}{r}54.83 \\
\pm 2.63\end{array}$ & $\begin{array}{c}102.17 \\
\pm 7.87^{*}\end{array}$ & $\begin{array}{c}44.67 \\
\pm 1.54^{*}, \mathrm{a}\end{array}$ & $\begin{aligned} & 86.00 \\
\pm & 3.01^{*}, \mathbf{b , c}\end{aligned}$ & $\begin{array}{l}87.50 \pm \\
3.42^{*, \mathbf{b}, \mathbf{c}}\end{array}$ & $\begin{array}{c}76.33 \\
\pm 4.28^{*}, \mathrm{a} \\
\end{array}$ & $\begin{array}{c}109.11 \\
\pm 3.18^{*}\end{array}$ & $\begin{array}{c}123.25 \\
\pm 2.94^{*}, \mathrm{a}\end{array}$ & $\begin{array}{c}118.33 \\
\pm 2.01^{*, a}\end{array}$ & & $\begin{array}{c}105.21 \\
\pm 2.81 *\end{array}$ & $\begin{array}{l}102.67 \\
\pm 1.96^{*}\end{array}$ \\
\hline $\begin{array}{c}\text { LDL-Ch } \\
\text { (mg/dl) }\end{array}$ & $\begin{array}{r}20.00 \\
\pm 1.24\end{array}$ & $\begin{array}{c}13.67 \\
\pm 0.42 *\end{array}$ & $\begin{array}{c}16.83 \\
\pm 0.65^{*, a}\end{array}$ & $\begin{array}{c}15.50 \\
\pm 0.43^{*, a}\end{array}$ & $\begin{array}{c}15.33 \\
\pm 0.67^{*}, \mathrm{a}\end{array}$ & $\begin{array}{c}15.83 \\
\pm 0.60^{*, a}\end{array}$ & $\begin{array}{c}12.50 \\
\pm 0.46^{*}\end{array}$ & $\begin{array}{c}11.77 \\
\pm 0.47 *\end{array}$ & $\begin{array}{c}13.33 \\
\pm 1.09 *\end{array}$ & $\begin{array}{c}13.36 \\
\pm 0.83 *\end{array}$ & $\begin{array}{c}13.93 \\
\pm 0.66^{*}\end{array}$ & $\begin{array}{c}13.33 \\
\pm 0.41^{*}\end{array}$ \\
\hline $\begin{array}{l}\text { HDL-Ch } \\
\text { (mg/dl) }\end{array}$ & $\begin{array}{r}24.50 \\
\pm 1.88 \\
\end{array}$ & $\begin{array}{c}54.33 \\
\pm 4.35^{*} \\
\end{array}$ & $\begin{array}{c}44.67 \\
\pm 1.54^{*, a} \\
\end{array}$ & $\begin{array}{c}47.83 \\
\pm 3.09^{*, \mathbf{b}} \\
\end{array}$ & $\begin{array}{c}45.83 \\
\pm 2.54^{*}, \mathbf{a} \\
\end{array}$ & $\begin{array}{c}37.33 \\
\pm 2.29^{*}, \mathbf{a} \\
\end{array}$ & $\begin{array}{c}49.30 \\
\pm 3.18^{*}\end{array}$ & $\begin{array}{c}57.83 \\
\pm 2.94 *\end{array}$ & $\begin{array}{c}61.27 \\
\pm 2.01 *\end{array}$ & $\begin{array}{c}54.04 \\
\pm 2.15^{*} \\
\end{array}$ & $\begin{array}{c}48.21 \\
\pm 2.81 *\end{array}$ & $\begin{array}{c}48.27 \\
\pm 1.96^{*}\end{array}$ \\
\hline
\end{tabular}




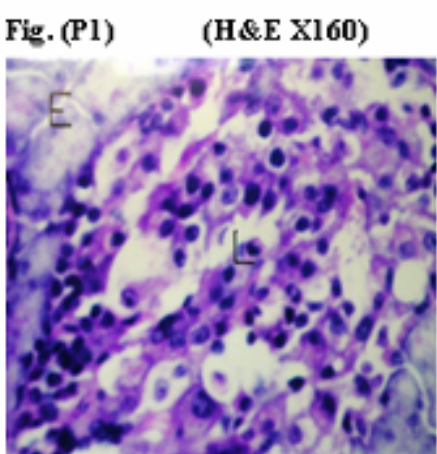

Pancreas of free control group showing normal histological structure of the cells in island of Langehans (L) and surrounding exocrine acini (E).

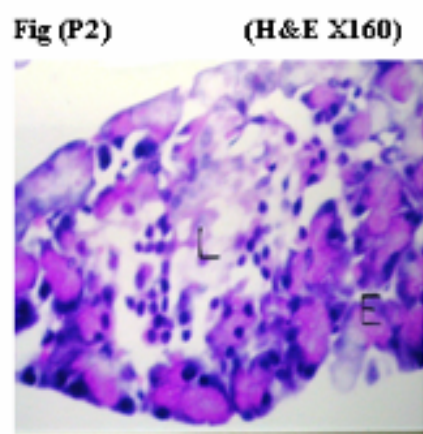

Pancreas of diabetic control group showing attophy in the cells of the island of Langehans (L)

Fig

(L1)

(H\&EX64)

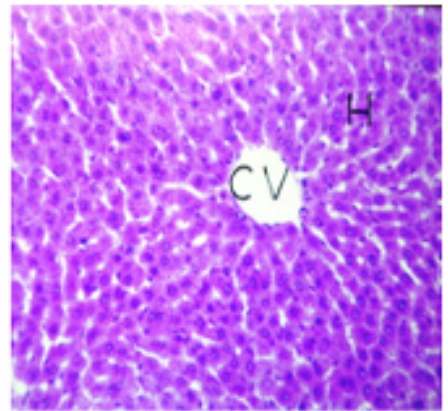

liver of the free control group showing normal histological structure of the central rein and surrounding hepatocytes (H) between the hepatocytes

Fig (L2)

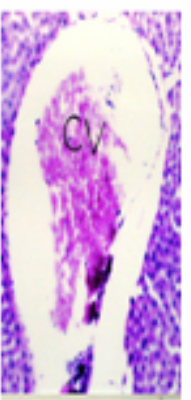

Liver of disbetic control group showing congestion in central vein (CV), in portal vein, cystic bile duct $(\mathrm{Bd})$ and preductal fibrosis
(H\&E X40)

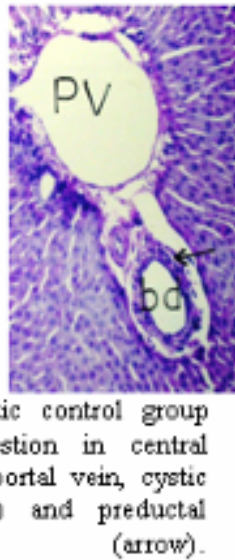

Fig (K1)

(H\&EX64)

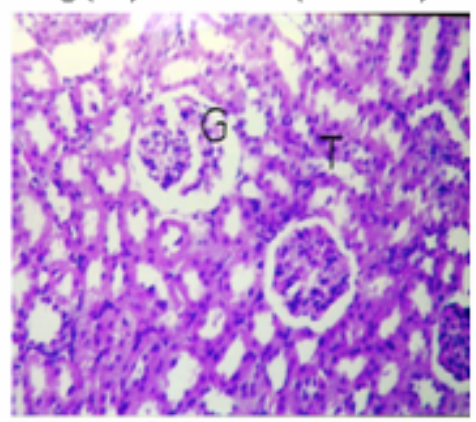

kidney of free control group showing normal histological structure of the glomeruli $(G)$ and surrounding tubules (T) at the cortex.
Fig (K2)

(H\&E X64)

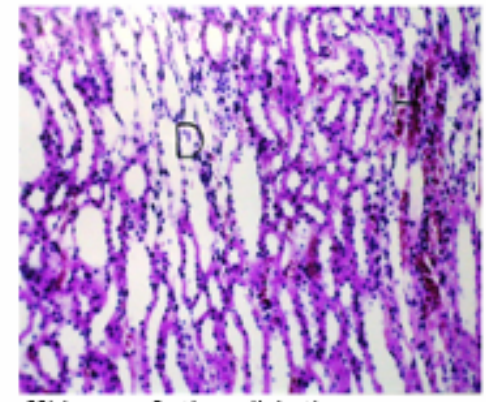

Kidney of the diabetic group showing focal degeneration in the tubules (D) and focal haemomages $(\mathrm{H})$ in the corticomedullary junction 
Fig (P3)

(H\&E X40)

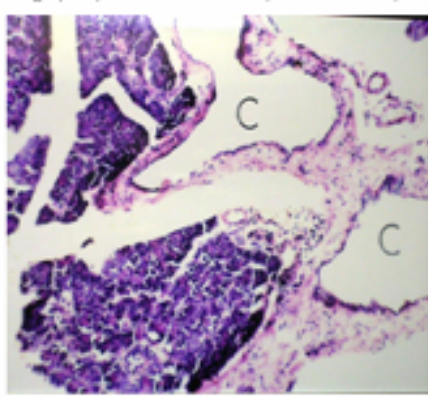

Pancreas of diabetic control group showing cystic dilatation of the duct system of the exocrine gland (C).
Fig (L3)

(H\&E X160)

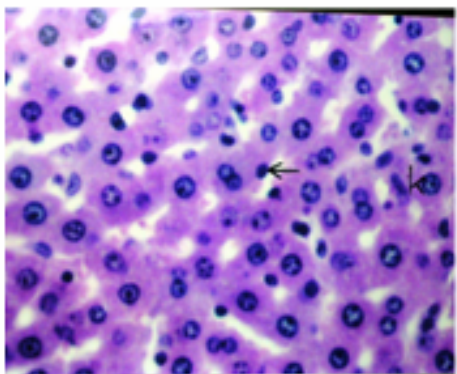

Liver of diabetic group showing mononuclear leucocytes inflammatory cells infiltration (arrow) and kupffer cells proliferation (k).
Fig(K3)

(H\&E X160)

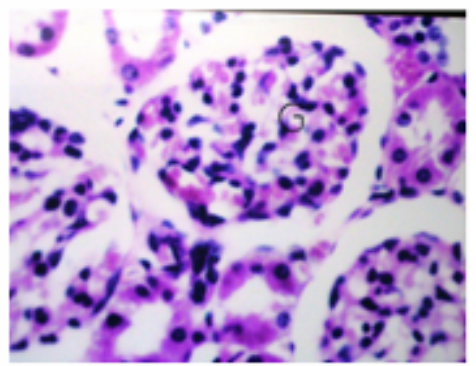

Kidney of the diabetic group showing swetling in the lining endothelial cells of the congested glomendi(G)
Fig (P4)

(H\&EXI60)

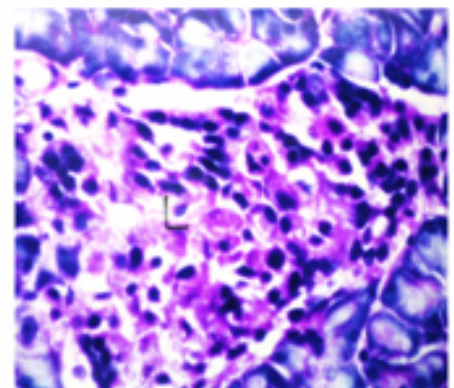

Pancreas of biphasic PPC treated group showing intact histological structure of the cells in island of Langerhans (L).
Fig (L4)

(H\&E X 160)

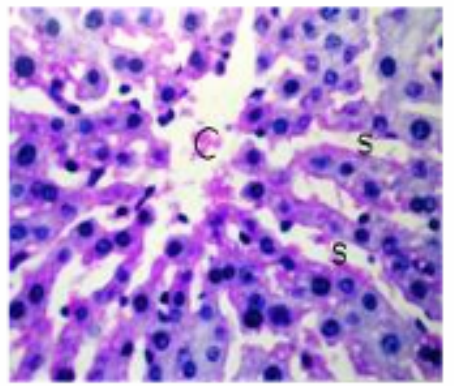

Liver of biphasic treated groups showing congestion in central vein (C) and blood sinusoids (S).
Fig (K4)

(H\&E Xl60)

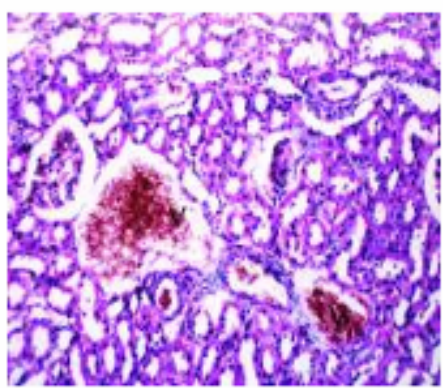

Kidney of biphesic treated groups showing congestion in cortical blood vessel (V). 
Fig (P5)

(H\&E X160)

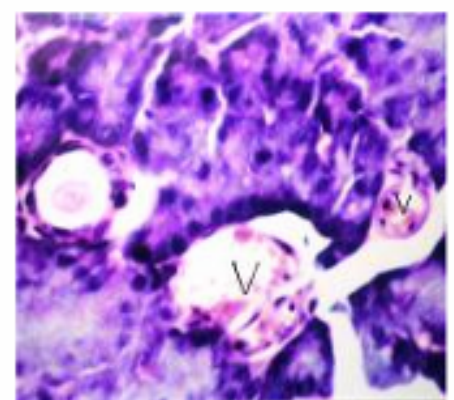

Pancreas of biphasic PC treated group showing congestion in the blood vessels (V).
Fig (L 5)

(H\&E X 64)

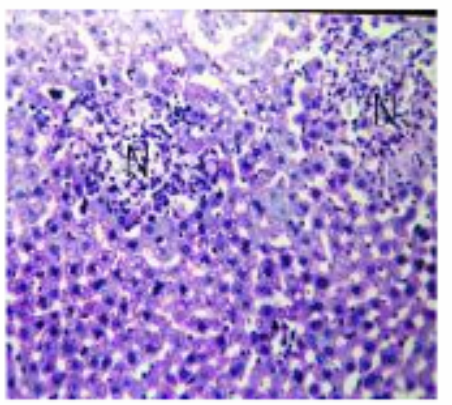

liver of regular treated groups showing focal necrosis in the hepatic parenchyma (N).

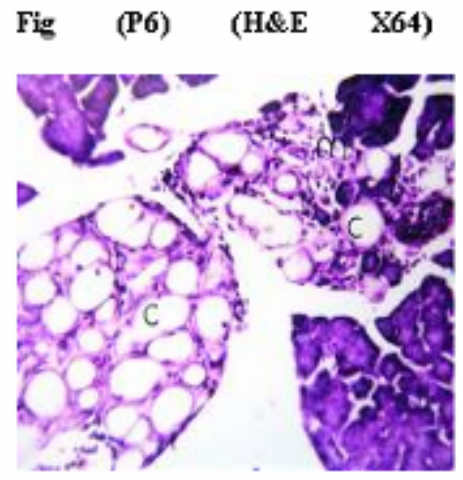

Pancreas of regular treated groups showing inflammatory cells infiltration (m) in the stroma between the cystic (C) exocrine ducts.
Fig (L6) (H\&E X160)

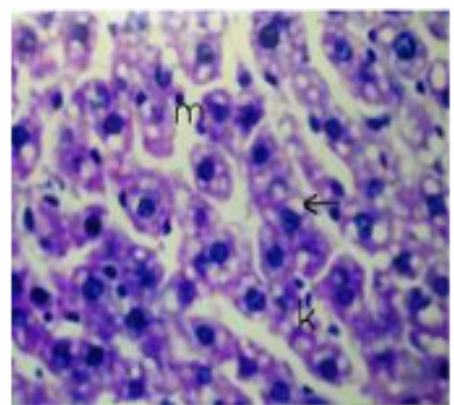

Liver of regular treated groups showing fatty change in the hepato-cytes (arrow), with mononucleas inflammatory cells infiltrations (m) and hupffer cells proliferations (k) in between the hepatocytes.
Fig (K5)

(H\&E X 160)

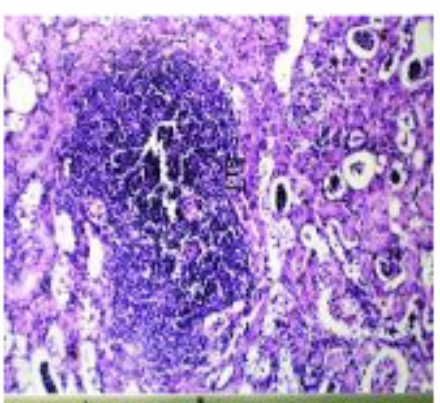

Kidney of regular treated groups showing focal suppurative embolus in the cortical portion (E)

Fig (K6)

(H\&E Xl60)

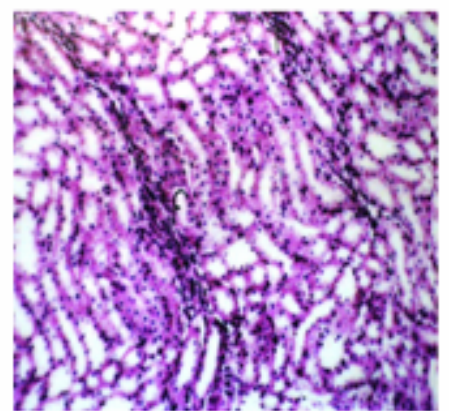

Kidney of regular treated groups showing focal fibrosis in corticomedullary junction (f). 


\section{DISCUSSION}

The aim of this study was to visualize the possible hypoglycemic effect of three INSUGEAST preps (PC, PPC \& SP) in IDDM and comparing it with that induced by insulin standard (ST). The study extended to evaluate the difference in effect between aforementioned preps in three different forms of action (regular, inside recombinant yeast cells and biphasic forms) against the stability and severity of hyperglycemia. The data showed that the hyperglycemic state of rats injected with INSUGEAST preps in biphasic form, showed marked improvement that increase with duration. In conform, the histopathological study of pancreas of these groups, observed no/little changes in island of Langerhans cells. In accordance with the study of Grossman et al. ${ }^{[23]}$, they evident that insulin treatment by implants or islet transplantation promotes $\beta$-cell regeneration in the STZ-diabetic mouse model of $\beta$-cell regeneration, and does so in a time dependent manner with longer treatment periods associated with greater recovery. They also suggested that the overall degree control of glycemic control affects regeneration with better control accelerating recovery of $\beta$-cell mass and function. Previous studies have shown that STZ-induced diabetes in rats is associated with vascular oxidative stress and dysfunction [24]. Recent study revealed that vascular dysfunction and oxidative stress strictly depend on insulin deficiency and are probably not associated with systemic toxic side effects of STZ ${ }^{[25]}$. On other hand, groups of rats treated with ST, SP showing partial improvement in the state of hyperglycemia. In consistent with the histopathological study, ST and SP injection still showing some atrophy and depletion in the cells of island Langerhans. To interpret the latter observations it was mentioned that insulin therapy does not diminished $\beta$ cells damage in neonatal STZ injected rats, but partial ß-recovery of islets of Langerhans was observed ${ }^{[26]}$. Insulin facilitates entry of glucose into muscle, adipose and several other tissues by facilitated of hexose transporters. GLUT4 are the major transporters are present in cytoplasmic vesicles. Binding of insulin to receptors on such cells leads rapidly to fusion of those vesicles with the plasma membrane and insertion of the glucose transporters, which used for glucose uptake in the plasma membrane ${ }^{[27]}$. In addition, Insulin stimulates the liver to store glucose in the form of glycogen. It activates the enzyme hexokinase, which phosphorylates glucose, trapping it within the cell. Coincidently, insulin acts to inhibit the activity of glucose6-phosphatase. Insulin also activates several of the enzymes that are directly involved in glycogen synthesis, including phosphofructokinase and glycogen synthase ${ }^{[28]}$. In contrast, the severity of hyperglycemia in rats treated with insulin either in regular form or in yeast cells was increased and the potent activity of insulin for glucose reduction was decreased. Also animals could not survive and 
mortality rate increased. These worst effects of insulin, might be due to that insulin action in regular form extend only for little hours of the day and so that rats in these groups subjected to hyperglycemia at the most hours of the day and behaved as poor control diabetes or insufficient short-acting insulin treatment. Concomitantly, the study of Ozlem et al. ${ }^{[29]}$ observed that the levels of hyperglycemia were higher in the STZ + insulin group than those in the STZ-only group. Histopathology was similar in the STZ and STZ + insulin groups, and degeneration was observed in both groups, but immunohistochemistry revealed a more severe reduction in insulin-secreting cells in the STZ + insulin group than that in the STZ group. So this study showed that insufficient short-acting insulin treatment can increase the diabetogenic effect of STZ in rats. In addition, insulin inside recombinant yeast cells might undergo successive degradation inside the alkaline media of the animal intestine and these degradations might be responsible for the morbidity and mortality of these animals. Confirming the biochemical observations, histopatholoical examination of the pancreas of these groups still showed atrophy in the cells of island and dilated ducts in addition to leucocytes inflammatory cells between them.

The $2^{\text {nd }}$ target of this study was to compare the possible protective effect of the 3 forms of tested INSUGEAST preps against the hepato-renal dysfunction and hyperlipidemia response to STZ injection. The data in this study declared impairment in liver enzymes by STZ injection and this showed by increasing the levels of liver enzymes (AST, ALT) as well as ALP. Concomitantly, it was observed that injection of STZ to rats was followed by massive impairment in the liver function and ALP ${ }^{[\mathbf{1 , 3 0}}$. The elevated levels of AST and ALT enzymes in STZ-diabetic rats are thought to be consisted with their greater need for gluconeogenic substrate and were attributed to induce synthesis of these enzymes [31]. Confirming the later biochemical observation, histopathological examination of rat liver injected with STZ showed obvious changes in liver structure. In agreement with the study of Ebrahim et al. ${ }^{[1]}$, it was found that STZ injection showed marked dilatation of the central vein, vacuolization and ballooning with disorganization of hepatocytes. Contrary, treatment with the INSUGEAST preps (in biphasic form) showed marked improvement of liver enzymes as well as in ALP. Moreover, data showed that insulin standard induced decreases in liver enzymes in the STZ-diabetic rats similar to that induced by these preps. Insulin was reported by many investigators to improve the enzymatic alteration in liver tissue in STZ induced diabetes $^{\left[{ }^{[32} \text {. Mechanism of insulin }\right.}$ action was through control the key enzymes of metabolism by phosphorylation or dephosphorylation of tyrosine residues. The insulin receptor is a tyrosine kinase, it functions as an enzyme that transfers phosphate groups from ATP to tyrosine residues on intracellular target proteins. Binding of insulin to the alpha subunits causes the beta subunits to phosphorylate themselves, 
thus activating the catalytic activity of the receptor. The activated receptor then phosphorylates a number of other intracellular proteins, in order to convert them to an active state. The resent study of Chen et al. ${ }^{[33]}$ showed that the capture of phosphoproteins by 14-3-3 proteins mediates actions of insulin. Furthermore it was showed that insulin-treated diabetic animals showed greater silymarin-induced hepatoprotection against antitubercular drugs-induced liver injury, which was characterized by near normal levels of marker enzymes, an increase in total proteins and normal hepatic structure to normal value ${ }^{[34,35]}$. In conform, the histopathological study on the liver of these groups showed mild improvement to the structural changes induced by STZ.

An additive worse effect in both liver enzymes and structure was recorded in rats treated with INSUGEAST preps in regular form. These are in consistent with the study of Ozlem et al. ${ }^{[2]}$, they showed that insufficient short-acting insulin treatment can increase the diabetogenic effect of STZ in rats, histopathology was similar in the STZ and STZ + INS groups, degeneration was observed in both groups, and immunohistochemistry revealed a more severe reduction in insulinsecreting cells in the STZ + INS group than that in the STZ group.

Another principal focus in this work was studying the therapeutic use of INSUGEAST preps against the toxic effects induced by STZ injection on the biochemical and structural changes in the kidney of treated groups. The elevation of both blood urea and serum Creat in the present study evident the nephrotoxic effect induced by STZ injection. In consistent with studies of Sjoquist et al. ${ }^{[36]}$; and Imaeda et al. ${ }^{[29]}$, they recorded impaired renal function after STZ injection. In addition the histopathological study of kidney removed from STZ diabetic rats showed marked changes in both glomeruli and tubules of kidney. Type 1 diabetes is considered as one of the most precipitating factors of renal failure it start with gloemerular affection and end, in poor control and chronicity, with tubular affections ${ }^{[37]}$. Treatment of STZ-diabetic rats with biphasic INSUGEAST especially PC showed marked improvement in kidney function. Several investigators showed that insulin can prevent impaired kidney function in STZ diabetic rats ${ }^{[1]}$ and it can used to regain hypertrophied kidney to its normal weight ${ }^{[38]}$. Furthermore, STZinduced diabetes causes impairment in calcium/calmodulin-dependent

contractile process of vas deferens, which is correctable partially following insulin therapy ${ }^{[39]}$. In addition to the biochemical analysis, mild improvement in kidney structure was observed by insulin preps. In contrast, worse effect in both kidney enzymes and structure was recorded in rats treated with INSUGEAST preps in regular form. These may be due to the insufficient short-acting insulin treatment, in the regular form, which can increase the diabetogenic effect of STZ in rats. Thus the resultant impairment hepato-renal function of these groups of regular form was secondary to/accompanied to the severity state of hyperglycemia. 
The present investigation was extended to test the effect of i.p injection of STZ on lipid metabolism and the possible ameliorative effect of INSUGEAST preps.

The dyslipidemia of these groups showed positive correlation with the severity of hyperglycemia. Mountains of researches were done, and all of which pointing to marked elevation in all fraction of lipid except HDL-Ch which showed negative correlation with the severity of the diabetes ${ }^{[40,41]}$. The hypolipidemic action of insulin was reports early by several investigators ${ }^{[42,43]}$, in which insulin is the $1^{\text {st }}$ not the $2^{\text {nd }}$ drug in treatment of hyperlipidemia in IDDM. Insulin promotes synthesis of fatty acids in the liver. When the liver is saturated with glycogen, any additional glucose taken up by hepatocytes is shunted into pathways leading to synthesis of fatty acids, which are exported from the liver as lipoproteins. The lipoproteins are ripped apart in the circulation, providing free fatty acids for use in adipocytes, which use them to synthesize triglyceride. Insulin inhibits lipase in adipose tissue that hydrolyzes triglycerides to release fatty acids. Insulin also facilitates entry of glucose into adipocytes, and within those cells, glucose can be used to synthesize glycerol. This glycerol, along with the fatty acids delivered from the liver, is used to synthesize triglyceride within the adipocyte. By these mechanisms, insulin is involved in further accumulation of triglyceride in fat cells ${ }^{[44]}$. From a whole body perspective, insulin has a fat-sparing effect. Not only does it drive most cells to preferentially oxidize carbohydrates instead of fatty acids for energy, insulin indirectly stimulates accumulation of fat in adipose tissue.

In conclusion the INSUGEA preps in biphasic form had the more potent effect in management of STZinduced IDDM not only synergist the hypoglycemic action of standard insulin, but also prevent the occurrence of most common series complication of diabetes (hepato-renal impairment and dyslipidemia). These effects were pronounced in $\mathrm{PC}$ and PPC preps and to a lesser extend in SP prep. This may encourage to advice to use these preps in Egyptian markets. Also we need to pay more attention to verify from the amount of protamine zinc in the preps and use insulin the biphasic form.

\section{Acknowledgements}

Author is grateful to Professor Dr. Adel B. Kholoussy, professor of pathology, Cairo University, for his histopathological study.

\section{REFRENCES}

1. Ibrahim, M. A. El-Agouza., Said, M. Rawy., Aida, M. Saad., Salwa, S. Lashin and Suzan, F. I. El-Sisi (2000): Possible ameliorative effect of sulfur containing amino acids on streptozotocin diabetic rats. $J$. Drug Res. Egypt 23 (2):213-223.

2. Briede, J., Stivrina, M., Stoldere, D.Z., Vigante, B. and Duburs, G. (2007): Effect of cerebrocrast, a new long-acting compound on blood glucose and insulin levels in rats when administered before and after STZ-induced diabetes mellitus. 
Cell Biochem. Funt. 25(6): 673680.

3. Myers, M.A., Laks, M.R., Feeneys, S.J. and Makey, I.R. (1998): Antibodies to ICA 512/IA-2in rodent models of IDDM. J. Autoimmun. 11(3):265272.

4. Koulmanda, M., Qipo, A., Chebrolu, S., Neil, J., Auchincloss, H. and Smith, R.N. (2003): The effect of low versus high dose of streptozotocin in cynomolgus monkeys (Macaca fascilularis). Am. J. Transplant. 3(3): 267-272

5. Palmino-Garibay, M.A., Revilla-Monsalve, C., CarenasSanchez, A., Polanco-Ponce, A.C. and Islas-Andrade, S. (1998): effect of induced diabetes on reproduction and development. Ginecol. Obstet. Mex. 66: 403-406.

6. Bennett, P.H. (1994): Definition, diagnosis, classification of diabetes and impaired glucose tolerance in: Joplin's diabetes mellitus. Kahn, C.R and Weir, G. C. editors $13^{\text {th }}$ eds. Lea \& Febiger, Philadelphia. Baltimor, Hong Kong, London, Monish, Sydney, Tokyo. Pp. 193-200.

7. Zagon, I.S., Sassani, J.W., McLaughlin, P.J. (2006): Insulin Treatment Ameliorates Impaired Corneal Reepithelialization in Diabetic Rats. Diabetic 55(4):1141-1147.

8. Filho, O.A.R. and Fazan, V.P.S. (2006): Streptozotocin induced diabetes as a model of phrenic nerve neuropathy in rats. Journal of Neuroscience Methods 151(2): 131-138.
9. Bixler, G.V., Vanguilder, H.D., Brucklacher, R.M., Kimball, S.R., Bronson, S.K., Freeman, W.M. (2011): Chronic insulin treatment of diabetes does not fully normalize alterations in the retinal transcriptome. $B M C$ Medical Genomics 4:40.

10. James E. F. (1996): Antidiabetic agents: Insulin In: Martindale The extrapharmacopia, $31^{\text {st }}$ eds., Pharmaceutical Press, London, P. 341.

11. Manoharan, C., Singh, J. (2009): Insulin loaded PLGA microspheres: effect of zinc salts on encapsulation, release, and stability. J. Pharm. Sci. 98, 529542 .

12. Ferrer-Miralles, N., DomingoEspin, J., Corchero, J., Vazquez, E., Villaverde, A. (2009): Microbial factories for recombinant pharmaceuticals. Microb. Cell Fact. 8, 17.

13. Bancrofet, J.D., Stevens, A. and Turner, D.R. (1996): "Theory and Practice of Histological Techniques" $4^{\text {th }}$ eds., Churchil Livingstone, New York, London, San Francisco, Tokyo.

14. Trinder, P. (1969): determination of blood glucose in blood using glucose oxidase, an alternative oxygen acceptor. Ann. Clin. Biochem. 6:24-28.

15. Reitman, S. and Frankel, S. (1957): A calorimetric method for glutamic pyruvate transaminase. Am. J. Clin. Path., 28:56-60.

16. Roy, A.V. (1970): Regular method for determination of alkaline phosphatase activity in serum with thymolphithlein 
monophosphate. Clin. Chem. 16(5): 431.

17. Patton, C. and Cruoch, S. (1977): Spectrophotometric and kinetics investigation of Bethelot Reaction for determination of ammonia. Anal. Chem., 49:464469.

18. Houot, O. (1985): Interpretation of Clinical Laboratory Tests. Etdited by Siest G., Henny J., Schiele F., Young D.S. Biomedical Puplication, 220-234.

19. Fossati, P. and Prencipe, L. (1982): The determination of triglyceride using enzymatic method. Clin. Chem., 28, 2077.

20. Allain, C.C., Poon, L.S., Chan, C.S.G., Richmonol, W., and Fu, P.C. (1974): Enzymatic determination of total serum cholesterol. Clin. Chem. 20(4):470.

21. Stein E.A. (1986): In Textbook of Clin. Chem. NW Tiez, Ed. WB Saunders, Philadelphia, Pp 879886.

22. Assmann, G., Jabs, H.U., Kohnert, U., Note, W. and Schriewer, H. (1984): Enzymatic determination of LDLcholesterol. Clin. Chem. Acta., 140, 77-83.

23. Grossman, E.J., Lee, D.D., Tao, J., Wilson, R.A., Park, S.Y., et al. (2010): Glycemic Control Promotes Pancreatic Beta-Cell Regeneration in StreptozotocinInduced Diabetic Mice. PLoS ONE 5(1): e8749, 1-6.

24. Zhang, L., Zalewski, A., Liu, Y., Mazurek, T., Cowan, S., Martin, J.L., Hofmann, S.M., Vlassara, H., Shi, Y. (2003): Diabetes-induced oxidative stress and low-grade inflammation in porcine coronary arteries. Circulation, 108:472-478.

25. Oelzea, M., Knorra, M., Schuhmachera, S., Heerena, T., Ottoa, C., Schulza, E., Reifenbergb, K., Wenzela, P., Münzela, T., Daibera, A. (2011): Vascular Dysfunction in Streptozotocin-Induced

Experimental Diabetes Strictly Depends on Insulin Deficiency. $J$ Vasc Res; 48 (4):275-284.

26. Calvo, R.M., Forcen, R., Obregon, M. J., Escobar-DelRey, F., Morreale-De-Escobar, G. and Regardera, J. (1998): Immunohistochemical and morphometric studies of the fetal pancreas in diabetic pregnant rats. Effect of insulin administration. Anat Rec. 251(2):173-80.

27. Bogan J. S. and Kandror K. V. (2010): Biogenesis and regulation of insulin-responsive vesicles containing GLUT4. Current Opinion in Cell Biology, 22:506512.

28. Bowen, R. (2009): Physiologic Effects of Insulin in: Hypertexts of Biomedical Science. Pathopysiology of the endocrine system by Laura Austgen, Melissa Rouge. Fort Collins, CO 80523, rbowen@colostate.edu.

29. Ozlem, O., Senay, T., Sima, S., Mahiye, O. (2007): Effect of Insufficient Insulin Treatment in Streptozotocin-Induced Diabetes Mellitus. Pancreas, 34 (3): 354358.

30. Imaeda, A., Kaneko, T., Aoki, T., Kondo, Y., and Nagase, $H$. (2002): DNA damage and the 
effect of antioxidants in streptozotocin-treated mice. Food Chem Toxicol 40: 979-987.

31. Rawi, S.M. (1995): Studies of the ability of sulpher containing compounds to block diabetogenic effect of alloxan in albino rats. Proc. Zool. Soc. A. R. Egypt, 26:244-259.

32. Rao, P.V., Pugazhhenthi, S., and Khandelwal, R.L. (1995): Insulin decreases the glycogen synthase-3 alpha m RNA levels by altering its stability in STZinduced rat liver. Biochem. Biophys Res Commun. 5; 217(1):250-256.

33. Chen, S., Synowsky, S., Tinti, M. and MacKintosh, C. (2011): The capture of phosphoproteins by 14-3-3 proteins mediates actions of insulin. Trends in Endocrinology and Metabolism. $\mathrm{xx}: 1-8$

34. Srivasta, R.K., Sharma, S., Verma, S., Arora, B., Lal, H. (2008): Influence of diabetes on liver injury induced by antitubercular drugs and on silymarin hepatoprotection in rats. Find Exp Clin Pharmacol, 30(10): 731 .

35. Atangwho, I.J., Ebong, P.E., Egbung, G.E., Uboh, F.E. and Utu-Baku, A.B. (2010): Extracts of Azadirachta Indica Can Modulate the Possible Hepatotoxic Effect of Vernonia Amygdalina in Diabetic and NonDiabetic Rats. J App Sci Res, 6(12): 2275-2279.

36. Sjoquist, M., Huang, W., Johansson, B. L. (1998): Effect of C-peptide on renal function at the early stage of experimental diabetes. Kidney Int. 54(3):758764.

37. Bojestig, M., Arngvist, H., and Karlber, B. (1996): Glycemic control and prognosis in type I diabetes patients with micro albuminuria. Diabetes Car. 19:313-320.

38. Rasch, R., and Dorup, J. (1997): Quantitative morphology of rat kidney during diabetes mellitus and insulin treatment. Diabetologia, 40 (7):802-809.

39. Öztürk, Y., and Aydın, S. (2006): Effects of streptozotocininduced diabetes and insulin on calcium responsiveness of the rat vas deferens. Life Sci. 78(10):1084-1090.

40. You, J.S., and Chang, k. J. (1998): Effect of taurine supplementation on lipid peroxidation, blood glucose and blood lipid metabolism in STZinduced diabetic rats. Adv. Exp. Med. Biol., 442:163-168.

41. Takaike, H., Uchigata, Y., Iwasaki, N., Iwamoto, Y. (2004): Transient elevation of liver tranaminase after starting insulin therapy for diabetic ketosis or kitoacidosis in newly diagnosed type 1 diabetes mellitus. Diabetes Res. Clin. Pract., 64(1):27-32.

42. Medow, M.S., Kletter, L.B., and Trachtman, H. (1994): Increased lipid fluidity in sunaptosomes from brains of hyper osmolal rats. Bioch Biophys. Acta, 1193 (2):323-329.

43. Masumi, Y., Hitoshi, K., Kouhei, K., Mikio, I. (2004): Combined effect of vitamin $\mathrm{E}$ and insulin on cataracts of diabetic 
rats fed a high cholesterol diet. Biological \& pharmaceutical bulletin, 27(3): 338-344.

44. Valverde, A.M., Benito, M., Lorenzo, M. (2005): The brown adipose cell: a model for understanding the molecular mechanisms of insulin resistance. Acta Physiol Scand, 183:59-73). 


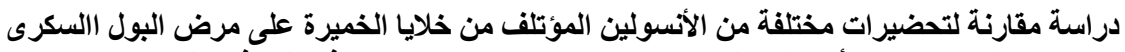
المعتمد على الأنسولين الناجم عن عقار الإستريبتوزوتوسين في الجرذانيان البيضاء

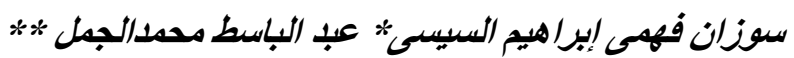

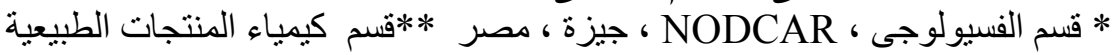
و الميكروبية ، المركز القومى للبحوث

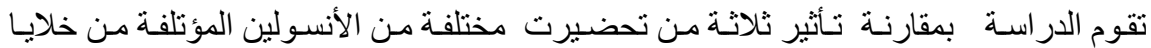

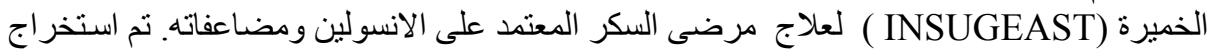

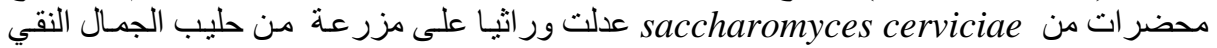

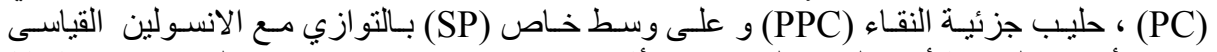

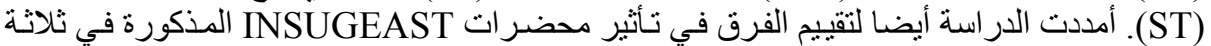

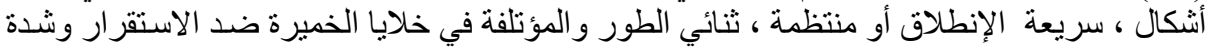

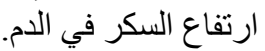

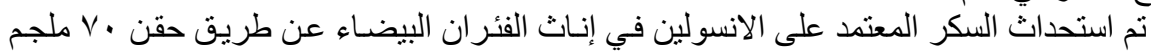

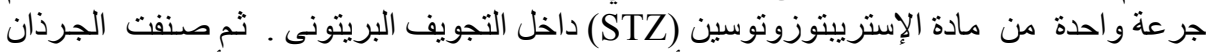

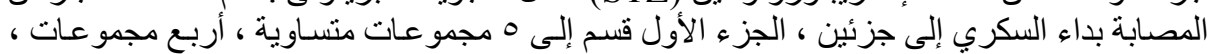

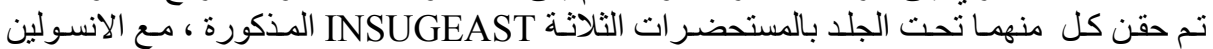

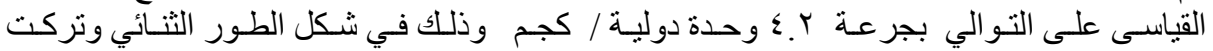

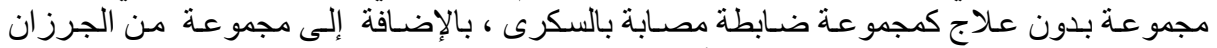

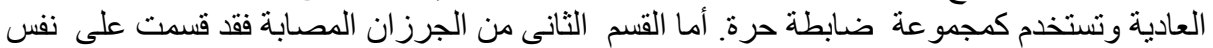

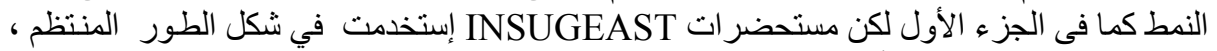

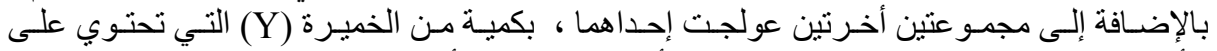

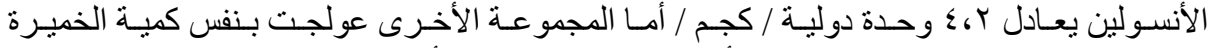

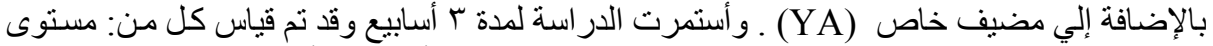

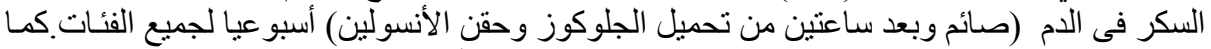

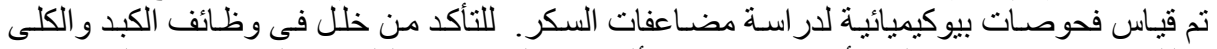

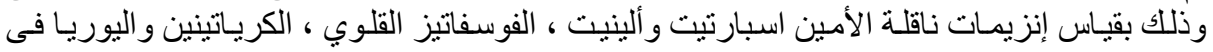

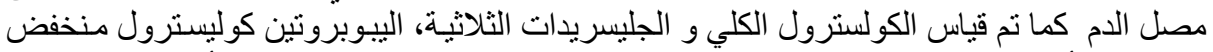

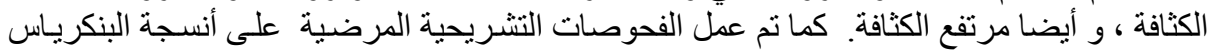

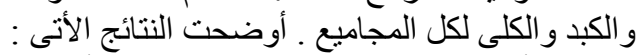

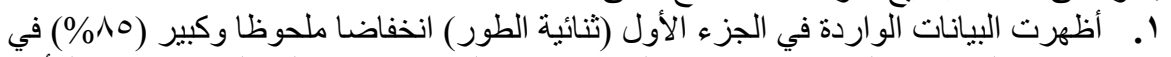

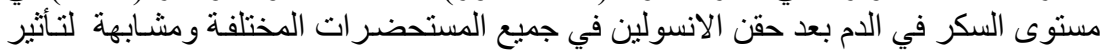

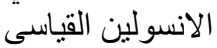

r r كما أظهرت تحسنا ملحوظا فى الخلل الوظيفى للكبد و الكلى و وأيضا الإرتفاع الدهنى، الناجمـة

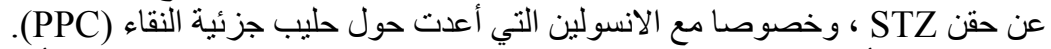

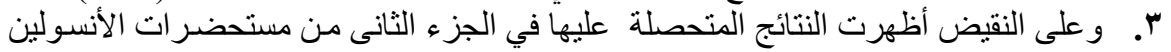

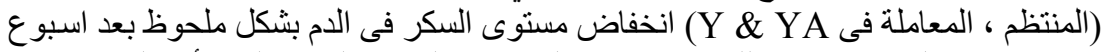

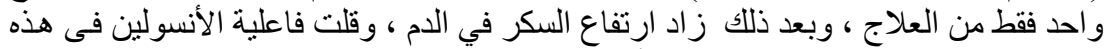

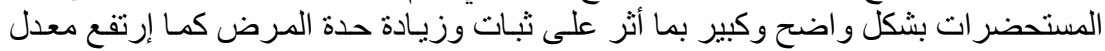

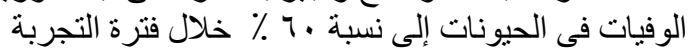


؛. ذادت مضـاعفات السكر (اختلال وظيفي للكبد و الكلى ، و إرتفاع الدهون) ولم تظهر أي ه. عززت الفحوصـات التشـريحية المرضـية على أنسـة البنكريساس والكبد و الكلى النتـائج البيوكيميائية المتحصل عليها.

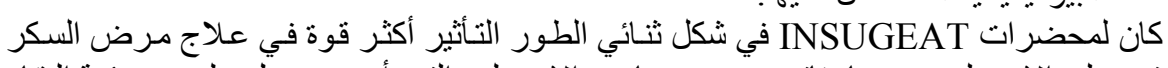

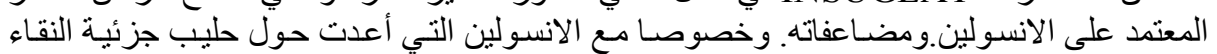

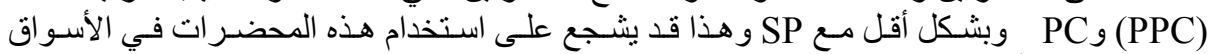

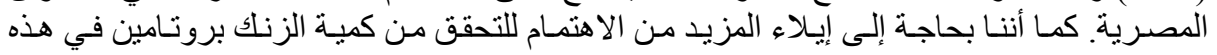

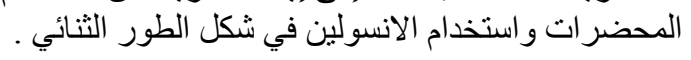

